\title{
Las asociaciones de pacientes y el control de la leishmaniasis en el Perú ${ }^{1}$
}

\author{
J. P. Guthmann, ${ }^{2}$ J. Calmet, ${ }^{3}$ E. Rosales, ${ }^{3}$ M. Cruz, ${ }^{3}$ J. Chang ${ }^{3}$ \\ y J. P. Dedet ${ }^{2}$
}

RESUMEN La leishmaniasis mucocutánea americana es un grave problema de salud en el Perú, particularmente en la región montañosa de Cuzco, donde se produjeron $25 \%$ de todos los nuevos casos notificados en 1989. El número de casos ha aumentado notablemente desde principios del decenio de 1980, cuando hubo una emigración estacional en gran escala a las zonas endémicas, especialmente a la región forestal de Madre de Dios, debido al descubrimiento de nuevas minas de oro en esa zona y al deterioro económico en el Perú. Ante la falta de respuesta oficial del Gobierno peruano, centenares de enfermos de leishmaniasis en la zona de Cuzco formaron asociaciones de autoayuda con el objetivo de obtener los medicamentos necesarios para tratar su afección. El logro principal de este movimiento que surgió espontáneamente, apoyado por varias instituciones públicas y privadas, fue animar a los enfermos, particularmente a los pacientes con lesiones de las mucosas, a salir del aislamiento. Como resultado, se han reducido mucho la prevalencia e incidencia de la enfermedad en ese territorio.

La leishmaniasis mucocutánea americana causada por Leishmania braziliensis es un grave problema de salud en el Perú. La incidencia anual de todas las manifestaciones clínicas de leishmaniasis americana aumentó de 7,6 por 100000 a 24,7 por 100000 en el período de 1979 a 1989 (Ministerio de Salud del Perú, datos inéditos, 1992). La leishmaniasis plantea un problema particular en la zona de Cuzco, donde

1 Se publicó en inglés en el Bulletin of the World Health Organization, Vol. 75, No. 1, 1997, con el título "Patients' associations and the control of leishmaniasis in Peru". (c) Organización Mundial de la Salud, 1997.

2 Facultad de Medicina de Montpellier, Laboratorio de Ecología Médica y Patología Parasitaria, Montpellier, Francia. Las solicitudes de separatas deben dirigirse a J. P. Guthmann a la siguiente dirección postal: MT Alexander von Humboldt, Universidad Peruana Cayetano Heredia, AP 5045, Lima 100, Perú.

3 Comité Regional para el Control de la Leishmaniasis en la Región Inca, Cuzco, Perú. se produjeron $25 \%$ de los casos nuevos notificados en 1989. En la actualidad, alrededor de $50 \%$ de todos los casos de leishmaniasis mucosa tratados en el Perú se notifican en esa zona. En el último decenio ha habido un aumento notable del número de casos nuevos tras una emigración estacional en gran escala a la zona forestal vecina de Madre de Dios, donde las compañías extractoras de oro emplean a pequeños agricultores durante la estación lluviosa. Sin embargo, la mayor prevalencia en ese territorio también ha sido el resultado de la falta de tratamiento por no disponerse de medicamentos apropiados. En 1983, los enfermos de leishmaniasis del poblado de Sicuani decidieron formar una asociación de pacientes a fin de conseguir medicamentos para tratar esa afección. Entre 1983 y 1993 se establecieron ocho asociaciones similares. En 1990, dichas asociaciones se unieron a las autoridades sanitarias y a otras instituciones del territorio para formar un comité que coordinara sus actividades.

En el presente artículo se describe la formación de esas asociaciones de pacientes y se discute su importancia para el control de la leishmaniasis mucocutánea. Hasta donde llegan nuestros conocimientos, en el campo de las enfermedades tropicales no hay indicios de otras iniciativas comunitarias en que los enfermos hayan podido organizarse espontáneamente y obtener los medicamentos necesarios para tratar su afección. En vista de la creciente prevalencia de leishmaniasis mucocutánea causada por L. braziliensis (1), su importancia para la salud pública y las dificultades del control (2), nuestras indagaciones pueden ser pertinentes para otros países donde esa forma de la enfermedad es endémica. 


\section{MATERIALES Y MÉTODOS}

\section{Desplazamiento de la población hacia Madre de Dios}

Aunque en la región de Cuzco ha habido actividades de extracción de oro desde el comienzo del presente siglo, la creciente ola de emigración hacia Madre de Dios comenzó en 1975, tras el descubrimiento de nuevas minas de oro y el marcado deterioro de la situación económica del Perú. Entre 1972 y 1977, la producción de oro aumentó de $122 \mathrm{~kg}$ anuales a $85 \mathrm{~kg}$ mensuales (3). La población abandonó sus comunidades en el altiplano, donde la situación socioeconómica había venido empeorando, para viajar a Madre de Dios en busca de trabajo en la industria de extracción de oro. La emigración se intensificó con el aumento de la demanda de trabajadores, a raíz de un incremento repentino de la demanda internacional de dicho metal. En una encuesta realizada en 1992 (J. Calmet, datos inéditos) se estimó que aproximadamente 35000 personas, sobre todo hombres jóvenes, emigran anualmente de diferentes zonas a Madre de Dios. En algunos lugares, la mitad de la población masculina de 15 a 35 años de edad emigra cada año. Casi todos esos emigrantes provienen de las zonas montañosas de la zona de Cuzco.

Madre de Dios, inmensa llanura de selva tropical, es un territorio donde la leishmaniasis causada por L. braziliensis es endémica (4). La enfermedad se transmite principalmente cuando se tala y quema la vegetación selvática antes de comenzar las actividades de extracción, pero también durante esas actividades, cuando la gente está expuesta a las picaduras de insectos flebótomos. Por lo general, las lesiones cutáneas tardan varios meses en sanar espontáneamente y no son tan graves como las de las mucosas que, en la mayoría de los casos, aparecen varios meses o años más tarde (5). El aumento del número de pacientes con lesiones de las mucosas se observó no en Madre de Dios, donde se contrae la infección, sino en las zonas montañosas de Cuzco cuando los trabajadores

CUADRO 1. Tasas de incidencia anual y número de casos de leishmaniasis notificados en el Perú, Cuzco y Madre de Dios en 1984, 1989 y 1991, respectivamente

\begin{tabular}{|c|c|c|c|c|c|c|}
\hline & \multicolumn{2}{|c|}{1984} & \multicolumn{2}{|c|}{1989} & \multicolumn{2}{|l|}{1991} \\
\hline & No. de casos & Incidenciaa & No. de casos & Incidencia ${ }^{a}$ & No. de casos & Incidencia ${ }^{a}$ \\
\hline Perú & 2733 & 14,1 & 5212 & 24,7 & 3631 & 16,5 \\
\hline Cuzco & 920 & 99,1 & 1297 & 127,0 & 722 & 69,0 \\
\hline Madre de Dios & 183 & 43,9 & 147 & 309,4 & 324 & 653,2 \\
\hline
\end{tabular}

Fuente: Oficina General de Epidemiología, Ministerio de Salud, Lima, Perú.

a Incidencia anual = No. de casos de leishmaniasis (en todas sus formas clínicas) por 100000 habitantes al año.

volvieron a su casa. La ausencia de insectos flebótomos en las montañas explica por qué la enfermedad no se transmite en esas zonas. Se produjo un aumento de la prevalencia de leishmaniasis en las áreas montañosas, donde la enfermedad de las mucosas se convirtió en un grave problema. Según los resultados de una investigación, en algunas zonas alrededor de 3\% de la población masculina se vio afectada por lesiones de las mucosas y esa proporción aumentó a $10 \%$ en hombres de 40 años (Centro de Investigación y Promoción Amazónica [CIPA], datos inéditos, 1991). El cuadro 1 muestra la elevada incidencia anual de leishmaniasis en las zonas de Cuzco y Madre de Dios en comparación con la incidencia general en el Perú.

En agosto de 1975 se declaró a la leishmaniasis una enfermedad ocupacional en el Perú y desde entonces el tratamiento ha sido gratuito para toda persona que la contraiga mientras trabaja en la selva. No obstante, el acceso al tratamiento era muy limitado antes de la creación de las asociaciones de pacientes.

\section{Metodología}

El estudio de la función de las asociaciones de pacientes en la zona de Cuzco se realizó entre enero y mayo de 1993. Había poca documentación sobre los pacientes y los principales métodos empleados para acopiar información eran las entrevistas informales y la recolección de datos de los registros de los hospitales y de las asociaciones de pacientes. Se entrevistó a los enfermos de leishmaniasis, a los dirigentes de las asociaciones de pacientes, a los trabajadores de salud y a las autoridades sanitarias. En general las entrevistas se realizaron en el lugar de trabajo o en la casa del entrevistado, se grabaron en cinta magnetofónica y se transcribieron. En algunos casos en que las personas solo hablaban quechua fue preciso trabajar con un intérprete. Se acopió información epidemiológica en los lugares donde habitualmente se examina a los pacientes (hospitales públicos, asociaciones de pacientes, instituciones privadas) y en el departamento de salud del territorio.

\section{RESULTADOS}

\section{Asociaciones de pacientes}

La primera asociación de pacientes de Cuzco se formó en 1983 por iniciativa de un grupo de personas con leishmaniasis que provenían del pueblo de Sicuani y que recorrieron el territorio pidiendo a quienes tuvieran leishmaniasis mucocutánea que se unieran a ellas: al cabo de algunos meses, más de 200 personas afectadas habían ingresado a la asociación. Contaban con el apoyo de varias instituciones, sobre todo de la Iglesia católica de la localidad, que divulgó información por medio de su radiodifusora local y les suministró medicamentos. Los pacientes se inscribieron y un médico viajó desde Cuzco para examinarlos; algunos fueron tratados con meglumina, pero los casos graves fueron enviados a hospitales en Lima y Cuzco para su tratamiento con amfotericina B. 
Entre 1983 y 1993 se estableció en el territorio un total de nueve asociaciones que representaban a 1648 miembros. La más grande, en el poblado de Ocongate, se estableció oficialmente en octubre de 1989, aunque sus actividades habían comenzado en febrero de 1988. Entre febrero de 1988 y enero de 1992, un total de 441 pacientes se habían inscrito en esa asociación. En el cuadro 2 se presenta la distribución anual de los casos según su forma clínica.

Conseguir medicamentos no era el único objetivo. La gente sostenía que el Gobierno debía obrar con mayor empeño: aunque legalmente la leishmaniasis se consideraba una enfermedad ocupacional, en la práctica no había medicamentos gratuitos ni se pagaba indemnización financiera a quienes la habían contraído en el trabajo. Los pacientes también exigían mejores condiciones de vida en Madre de Dios y que las compañías de extracción de oro establecieran normas mínimas para las condiciones laborales. Otros objetivos de la asociación, como la búsqueda de oportunidades adicionales de empleo estacional para evitar que la gente emigrara a la selva, no se relacionaban directamente con la leishmaniasis.

Cada asociación de pacientes elige a un directorio cada 2 años y en tres o cuatro ocasiones al año se celebran reuniones que duran todo un fin de semana y que ofrecen la oportunidad de discutir varios aspectos de la enfermedad y la emigración. Todos los pacientes son examinados por un médico especialista en enfermedades tropicales. La mayoría de las personas no necesitan tratamiento y asisten a la reunión siguiente para hacerse otro reconocimiento. Las personas que necesitan meglumina son tratadas por un trabajador del centro de salud; los pacientes que viven en zonas aisladas son atendidas por el promotor de salud de la comunidad. Los individuos que necesitan tratamiento con amfotericina B son remitidos al hospital de Cuzco. Se ofrece educación general para adultos, además de tratamiento para la enfermedad y otros problemas de salud. También se organizan cursos de capacitación y actividades de información. Aunque los problemas de salud son
CUADRO 2. Número de casos de leishmaniasis registrados en la asociación de pacientes de Ocongate (zona de Cuzco) entre febrero de 1988 y enero de 1992

\begin{tabular}{|c|c|c|c|c|}
\hline \multirow[b]{2}{*}{ Fecha } & \multicolumn{3}{|c|}{ No. de casos de cada forma clínica: } & \multirow[b]{2}{*}{ Total } \\
\hline & Lesión cutánea & Lesión de las mucosas & Cicatriz & \\
\hline Febrero 1988 & 13 & 44 & 28 & 85 \\
\hline Enero 1989 & 6 & 15 & 6 & 27 \\
\hline Febrero 1989 & 20 & 18 & 28 & 66 \\
\hline Enero 1990 & 16 & 27 & 13 & 56 \\
\hline Febrero 1990 & 13 & 20 & 19 & 52 \\
\hline Enero 1991 & 10 & 11 & 15 & 36 \\
\hline Febrero 1991 & 17 & 20 & 37 & 74 \\
\hline Enero 1992 & 20 & 15 & 10 & 45 \\
\hline Total & 115 & 170 & 156 & 441 \\
\hline
\end{tabular}

Fuente: M. Tokushima, Centro de Investigación y Promoción Amazónica, Cuzco, Perú. los más importantes, también se discuten asuntos sociales y políticos, tales como las condiciones de trabajo o de vida en la selva y la inobservancia de la legislación ocupacional por los propietarios de las compañías mineras.

En cuanto a los casos detectados y tratados, los resultados más importantes se han obtenido en Ocongate. En 1992 se trató a un total de 93 pacientes y se registraron menos de 10 casos nuevos de leishmaniasis mucosa en las asociaciones de pacientes. Un registro pormenorizado de los pacientes también ha suministrado datos epidemiológicos precisos. Por ejemplo, en el cuadro 3 se presenta una descripción clínica de todos los casos de leishmaniasis registrados en la asociación de pacientes de Ocongate hasta diciembre de 1992. Se evaluó el intervalo transcurrido entre la infección y la aparición de lesiones en las mucosas en 180 de esos pacientes. En la mayoría de los casos ese intervalo fue de 1 a 10 años, pero en un caso el lapso fue de 37 años y en nueve, de menos de 1 año. Se evaluó el seguimiento al cabo de 1 año en 239 casos: $163(68 \%)$ se curaron después del tratamiento con compuestos de antimonio pentavalente, pero varios pacientes sufrieron recaída. Solo cinco pacientes de Ocongate abandonaron el tratamiento.

\section{El comité regional}

Después de su establecimiento, las asociaciones de pacientes fueron apo-
CUADRO 3. Características clínicas de los casos de leishmaniasis registrados en la asociación de pacientes de Ocongate hasta diciembre de 1992

\begin{tabular}{lcc}
\hline \multicolumn{1}{c}{ Característica clínica } & No. de casos & Proporción (\%) \\
\hline Localización de la lesión & 91 & \\
Cara o cuello & 108 & 18,9 \\
Tronco o extremidades superiores & 282 & 22,4 \\
Extremidades inferiores & & 58,6 \\
No. de lesiones & 351 & \\
$1-2$ & 145 & 70,7 \\
$3-10$ & & 29,3 \\
Forma clínica & 270 & 54,4 \\
Lesión cutánea solamente & 226 & 45,6 \\
Afección de la mucosa & 64 & 12,9 \\
Afección de la laringe & 64 & \\
\hline
\end{tabular}

Fuente: M. Tokushima, Centro de Investigación y Promoción Amazónica, Cuzco, Perú. 
yadas por las parroquias católicas de Sicuani y Ocongate, el Centro de Investigación y Promoción Amazónica (organización nacional no gubernamental), el Programa de Salud Comunitaria en el Trapecio Andino (grupo italiano de cooperación), el Instituto de Medicina Tropical de la Universidad Cayetano Heredia de Lima, el Instituto Peruano de Seguridad Social y el Ministerio de Salud. En 1990, esas instituciones establecieron un comité para la coordinación del control de la leishmaniasis en la zona de Cuzco, que más tarde recibió el apoyo de las autoridades de salud regionales y nacionales. El movimiento, que fue lanzado como iniciativa espontánea, quedó mejor estructurado y organizado. Se determinó el papel de cada institución y se definió la estrategia de control sobre la base de la búsqueda activa de casos, el diagnóstico precoz y el tratamiento inmediato de la enfermedad. Dicha iniciativa fue similar a la de Bolivia, aunque se puso en práctica de otra manera (6).

\section{DISCUSIÓN}

Cuando la prevalencia de una enfermedad es elevada en una población, es preciso facilitar el tratamiento en un centro de salud o un hospital y los pacientes deben poder llegar al establecimiento asistencial. En el caso de la leishmaniasis mucocutánea en la zona de Cuzco en el Perú, no existía ninguna de esas condiciones a comienzos de los años ochenta, cuando se estableció la primera asociación de pacientes. El suministro de medicamentos contra la leishmaniasis no era suficiente y los pacientes se quedaban en sus comunidades y se negaban a ir a los hospitales y centros de salud, en la creencia de que ahí no podrían tratarlos. Los pacientes trataban sus lesiones cutáneas con medicinas tradicionales, a menudo ineficaces, o con ampollas de meglumina compradas en el mercado negro o en farmacias locales. En ambos casos, las lesiones cutáneas persistían varias semanas o meses y una elevada proporción de los pacientes presentaban lesiones de las mucosas. Los pacientes estaban distribuidos por todo el territo- rio y a menudo vivían en comunidades muy alejadas de los pueblos más grandes y a las que era difícil llegar. Muchos consideraban que su enfermedad era incurable. Además de la falta de tratamiento, la ignorancia del personal de salud también había contribuido a la acumulación de casos con lesiones mucosas. La formación de asociaciones de pacientes fue un movimiento que nació espontáneamente de las personas afectadas por la enfermedad como consecuencia de esa situación.

El principal logro de las asociaciones de pacientes, particularmente en Sicuani y Ocongate, fue animar a los enfermos a salir de su aislamiento. Aunque el movimiento fue apoyado por otras instituciones, fueron los pacientes quienes decidieron organizarse. Viajaron a Lima y Cuzco a reunirse con las autoridades sanitarias y recorrieron sus comunidades en busca de otros pacientes. Una vez establecidas las asociaciones, la participación de los pacientes tuvo importancia a la hora de administrar y supervisar el tratamiento en la comunidad. Esto quizá explica los resultados alcanzados en Ocongate en relación con el gran número de casos detectados y tratados, las tasas reducidas de incumplimiento y el acopio de datos epidemiológicos precisos. En la actualidad, muchos años después de la formación de las primeras asociaciones de ese tipo, centenares de pacientes siguen acudiendo a las reuniones que se celebran varias veces al año. Al parecer no hay actividades similares en otros países con leishmaniasis endémica (7).

Es importante entender las razones del éxito especial que han tenido las asociaciones aquí descritas. Obviamente, la explicación no radica en la concentración de un gran número de casos de leishmaniasis en una zona pequeña, ya que la prevalencia de otras enfermedades, como la malaria o la tuberculosis, también es elevada en esa zona. Una razón más importante es quizá la gravedad de las lesiones de las mucosas y su efecto en las personas. No es raro observar dificultades para respirar y hablar y la ingestión de alimentos se convierte en una actividad dolorosa, fenómeno que lleva a la malnutrición. La comunidad trata a los pacientes como personas inútiles y los vecinos y la familia los rechazan. A la larga, los pacientes se avergüenzan y deprimen, se esconden por años en sus comunidades y a veces terminan por suicidarse. Con el apoyo de varias instituciones públicas y privadas, las asociaciones de pacientes lograron introducir, incluso en el ámbito local, el uso de los últimos protocolos de tratamiento, otro factor importante que contribuyó al éxito. Las características sociales de la población destinataria también desempeñaron un papel decisivo. Los agricultores quechuas son un grupo social que ya se habían organizado en movimientos políticos y con mucha experiencia en la defensa de sus derechos frente a los terratenientes adinerados de la zona. Cuando se formaron las asociaciones de pacientes, los pobladores de las comunidades con leishmaniasis fueron invitados a hacerse socios por personas de características similares que padecían de la misma enfermedad, es decir, por pequeños agricultores quechuas semejantes a ellos y que vivían en condiciones similares. No les repugnaban las lesiones, como sucedía a menudo con personas de la población en general y el personal de los servicios de salud, que creían que la enfermedad era contagiosa. La relación particular entre los pacientes también ha ejercido influencia en los resultados logrados; el apoyo psicológico de las asociaciones ha sido inmenso.

La función del comité regional también ha revestido importancia. Todas las instituciones interesadas habían trabajado sobre el terreno muchos años, tenían vínculos estrechos con la población local y conocían detalladamente los campos de interés y sus problemas. Por ende, estaban en la mejor posición para determinar cuáles eran las intervenciones más adecuadas y escoger las actividades de control más apropiadas. Se acordó que la complejidad de la afección exigía la aplicación de un esquema de control de tipo general. Se determinó, por ejemplo, que era preciso tener activo un sistema de detección de casos, puesto que la gente se mostraba renuente a acudir espontáneamente al 
centro de salud en esa zona particular del país. Se examinó la posibilidad de impedir que las personas emigraran a Madre de Dios, pero se llegó a la conclusión de que esa medida era poco realista, puesto que para muchas personas la emigración estacional es la única forma de aumentar sus ingresos. Los factores económicos tampoco son la única causa de emigración, y por lo tanto mejorar la situación económica local no sería la manera de evitarla.

Cabe señalar, por último, que en este proceso las instituciones y autoridades de salud han trabajado juntas para sentar una política pertinente. Al menos, esto ha sucedido en el caso de las autoridades de salud regionales, con quienes se ha colaborado estrechamente en muchas de las actividades realizadas. Sin embargo, las autoridades nacionales a menudo se han mostrado renuentes a aceptar que existe el comité regional. La experiencia del $\mathrm{Co}^{-}$ mité regional es un ejemplo de la forma en que las organizaciones gubernamentales y no gubernamentales pueden trabajar juntas para formular una estrategia en que ni la intervención exclusiva del gobierno ni la de la población civil daría buenos resultados.

\section{CONCLUSIONES}

Al comienzo de los años ochenta, las personas afectadas por la leishmaniasis en la zona de Cuzco, Perú, carecían de acceso al tratamiento y muchos profesionales de salud que trabajaban en los hospitales y centros de salud del territorio sabían poco sobre esa afección. Al cabo de 10 años habían cambiado muchas cosas. En 1985 se incluyó la leishmaniasis en el Programa Nacional de Control de Enfermedades Tropicales, que hasta entonces cubría solo la malaria y la fiebre amarilla. En 1993, el Ministerio de Salud definió las normas para el control de la leishmaniasis y se lanzó una campaña regional de diagnóstico y tratamiento financiada por la OPS. Sin embargo, muchos pacientes siguen sin tratamiento a pesar de los avances alcanzados.

La comunidad ha desempeñado un papel fundamental en lograr que se efectuaran estos cambios, primero con la formación de sus propias asociaciones de pacientes y más tarde con la coordinación de sus actividades mediante un comité constituido por representantes de diversos sectores de la sociedad, entre ellos las autoridades sanitarias. Aun a sabiendas de que la experiencia de Cuzco no es aplicable en todas las situaciones, creemos que es un ejemplo de fructífera coordinación multisectorial y participación comunitaria que puede ser pertinente en muchos países donde la leishmaniasis mucocutánea es endémica.

Agradecimiento. Agradecemos a las autoridades nacionales de salud del Perú que nos hubieran permitido realizar esta investigación y el apoyo técnico brindado por las autoridades regionales de salud y las instituciones del Comité Regional para el Control de la Leishmaniasis. También manifestamos nuestra gratitud a toda la población de la zona de Cuzco que accedió amablemente a dejarse entrevistar, en particular a los miembros de las asociaciones de pacientes de Ocongate y Sicuani.

\section{REFERENCIAS}

1. World Health Organization. Control of the leishmaniases: Report of a WHO Expert Committee. Geneva: WHO; 1990. (WHO Technical Report Series No. 793).

2. Desjeux R. Human leishmaniasis: Epidemiology and public health aspects. World Health Stat Q 1992;45:265-267.

3. Aznar P, Luna M. Estudio de mineros migratorios que extraen oro en el Departamento de Madre de Dios. Lima: Centro de Investigación y Promoción Amazónica y Ministerio de Trabajo y Promoción Social; 1977.

4. Dedet JP. Leishmania et leishmanioses du continent américain. Ann Inst Pasteur 1993; 1:3-25.

5. Marsden PD. Mucosal leishmaniasis ("Espundia" Escomel, 1911). Trans R Society Trop Med Hyg 1986;80:859-876.

6. Dedet JP, Melogno R, Cárdenas F, Valda L, David C, Férnandez V, et al. Rural campaign to diagnose and treat mucocutaneous leishmaniasis in Bolivia. Bull World Health Organ 1995;73: 339-345.

7. Manderson L, Valencia L, Thomas B. Bringing the people in: community participation and the control of tropical diseases. Geneva: World Health Organization; 1991. (Documento inédito WHO TDR/SER/RP/92.1).

ABSTRACT American mucocutaneous leishmaniasis is an important health problem in Peru, particularly in the mountainous Cuzco Region, where 25\% of all new cases reported in 1989 were located. Cases have increased considerably since the beginning of the 1980s, when large-scale seasonal migration to endemic zones occurred, particularly in the forest area of Madre de Dios, following the discovery of new gold deposits there and the deterioration in the economic situation in Peru. Following the lack of official response from the Peruvian government, hundreds of people suffering from leishmaniasis in the Cuzco area formed self-help associations with the objective of obtaining the drugs needed to treat their disease. The major achievement of this spontaneous movement, which was supported by several public and private institutions, was to encourage sick people, particularly patients with mucosal lesions, to emerge from isolation. As a result, the prevalence and incidence of the disease have now considerably decreased in the region. 